\title{
Transillumination and HDR Imaging for Proximal Caries Detection
}

Journal of Dental Research 2018, Vol. 97(7) 844-849

(C) International \& American Associations for Dental Research 2018 Reprints and permissions: sagepub.com/journalsPermissions.nav DOI: 10.1 | 77/00220345/8759957

journals.sagepub.com/home/jdr

\author{
A. Lederer', K.H. Kunzelmann', R. Hickel', \\ and F. Litzenburger'
}

\begin{abstract}
The purpose of this study was to develop an in vitro model for the validation of near-infrared transillumination (NIRT) for proximal caries detection, to enhance NIRT with high-dynamic-range imaging (HDRI), and to compare both methods, using micro-computed tomography $(\mu \mathrm{CT})$ as a reference standard. Both proximal surfaces of 53 healthy or decayed permanent human teeth were examined using the Diagnocam (DC) (KaVo) and NIRT with HDRI (NIRT-HDRI). NIRT was combined with HDRI to improve the diagnostic performance by reducing under- and overexposed image areas. For NIRT-HDRI, an exposure series was captured and merged into a single HDR image. A classification was applied according to lesion depth. All surfaces were assessed twice by 2 trained examiners, and additionally with $\mu \mathrm{CT}$ for validation. The Kappa statistic was used to calculate inter-rater reliability and agreement between DC and NIRT-HDRI. Inter-rater reliability (weighted Kappa, wK) showed very good agreement for the DC (0.90) and NIRT-HDRI (0.96). The overall agreement (wא) was almost perfect (0.85). In the individual categories (0 to 4), the agreement (simple Kappa) ranged from almost perfect (category 4 ) to moderate ( $I$ and 2 ) to substantial (categories 0 and 3 ). Sensitivity and specificity of sound surfaces, enamel, and dentin caries ranged from 0.57 to 0.99 and were similar for both methods in the different categories. NIRT-HDRI had a higher sensitivity for sound surfaces and enamel caries, as well as a higher specificity for dentin caries. Regarding the obtained images, HDRI allowed for the detection of caries within a greater range of luminance levels, resulting in a more detailed visualization of structures without underor overexposure. However, HDRI this did not improve the diagnostics significantly. Distinguishing between a processed demineralized enamel and dentin lesions appears to be a problem specific to NIRT and cannot be balanced using HDRI.
\end{abstract}

Keywords: near-infrared light, dental caries, diagnostic imaging, high dynamic range imaging, X-ray microtomography, image enhancement

\section{Introduction}

The detection of proximal caries at an early stage is a major challenge. Visual-tactile and radiographic examination have their limitations and are insufficient for the detection of incipient proximal lesions in many cases. Visual assessment is difficult in the interdental space and the prevalence of proximal lesions are therefore underestimated (Poorterman et al. 1999; Poorterman et al. 2002). Radiographs often show overlapping effects and artifacts, and their sensitivity for the detection of enamel caries is moderate (Abesi et al. 2012). Furthermore, repeating radiographs is restricted because of ionizing radiation. Therefore, additional diagnostic methods of sufficient diagnostic accuracy are needed for the early detection of proximal lesions (Hume 1993; Featherstone 1999; National Institutes of Health 2001).

Diaphanoscopy has been applied in different fields of general medicine for many years, even before the discovery of X-rays (Kierzek 1995; Shurtleff et al. 1966). In dentistry, transillumination is also a highly promising method for caries detection. Following the development of fiber optics using visible light, the focus has shifted toward the use of near-infrared (NIR) light for the transillumination of teeth (Bussaneli et al. 2015; Kachalia 2015; Simon et al. 2015; Kühnisch et al. 2016; Simon et al. 2016).
Sound enamel is highly transparent when imaged in the infrared range. As such, irregularities, such as demineralization, can be visualized with high contrast (Jones et al. 2003; Fried et al. 2005). Several diagnostic tools using NIR light-for example, the Diagnocam (DC) (KaVo) or the VistaCam (Dürr Dental)- have been introduced to the dental market over the past $5 \mathrm{y}$.

Various clinical studies have discussed and assessed the utility of DC (Abdelaziz and Krejci 2015; Abdelaziz et al. 2016; Kühnisch et al. 2016). Two NIR laser diodes (with a power of $1 \mathrm{~mW}$ and a wavelength of $780 \mathrm{~nm}$ ) transilluminate molars and premolars from the buccal and lingual sides through the gingiva, alveolar process, and dental roots up to the crown. A monochrome complementary metal-oxide-semiconductor (CMOS) sensor captures the images from the occlusal direction

'Department of Operative Dentistry and Periodontology, University Hospital, LMU Munich, Germany

Corresponding Author:

A. Lederer, Department of Operative Dentistry and Periodontology, University Hospital, LMU Munich, Goethestrasse 70, 80336 Munich, Germany.

Email: aleder@dent.med.uni-muenchen.de 
with an image angle of $105^{\circ}$. Live images can be visualized and captured on a computer.

Several in vivo studies have suggested the potential of this technique for the detection of proximal caries, suggesting comparable or even superior clinical performance over digital bitewing radiographs. However, in clinical use, DC images often have under- and overexposed areas. This may be a result of intense light sources in the environment that cause reflections, metallic restorations in the examined area, or direct laser light into the camera due to a lack of adaptation. Hypo- or hypermineralization, or the morphology of the occlusal surface can also have an effect. In cases where the brightness difference in a picture from light to dark areas is higher than the camera sensor can display, high-dynamic-range imaging (HDRI) can be useful. Differently exposed images are needed to calculate a HDR image, allowing for a greater range of luminance levels and evenly exposed, sharper and more detailed images. The combination of NIR transillumination (NIRT) with HDRI may offer an avenue to improve NIRT image quality.

DC has been used in several in vitro studies (MarinovaTakorova et al., 2014; Abdelaziz et al., 2016; Abogazalah et al., 2017). However, our in vitro model differs significantly from those previously presented. When coherent laser light is directly applied on extracted teeth, speckle patterns are produced, causing poor image quality. At present, there is no solution to reduce the speckle phenomenon or under- and overexposed image areas; this is emphasized in the development of our in vitro model.

The purpose of this in vitro study was to develop an in vitro model for the validation of NIRT for proximal caries detection, to enhance NIRT with HDRI (NIRT-HDRI), and to compare both methods using micro-computed tomography $(\mu \mathrm{CT})$ as a reference standard.

\section{Materials and Methods}

\section{Sample Selection}

Fifty-three freshly extracted healthy or decayed permanent human teeth with fully formed apices were selected by 2 examiners (A.L. and F.L.). The specimens showed either an intact, sound interproximal surface or non-cavitated proximal caries lesions. An equal number of intact and visibly changed proximal surfaces was obtained. Teeth with metallic restorations, secondary caries, residual caries or damage due to the extraction process were excluded from this study. The selected teeth were cleaned with an acoustic scaler to remove plaque, calculus, and debris, and stored in distilled water at $4^{\circ} \mathrm{C}$. Age, sex, and race of the patients were unknown. All experimental procedures were approved by the Ethics Committee of the Medical Faculty, Ludwig Maximilians University in Munich, Germany (488-15 UE).

\section{Examination by NIRT}

An in vitro model was developed to simulate the working principle of the DC in clinical use (Figure 1). NIR light is

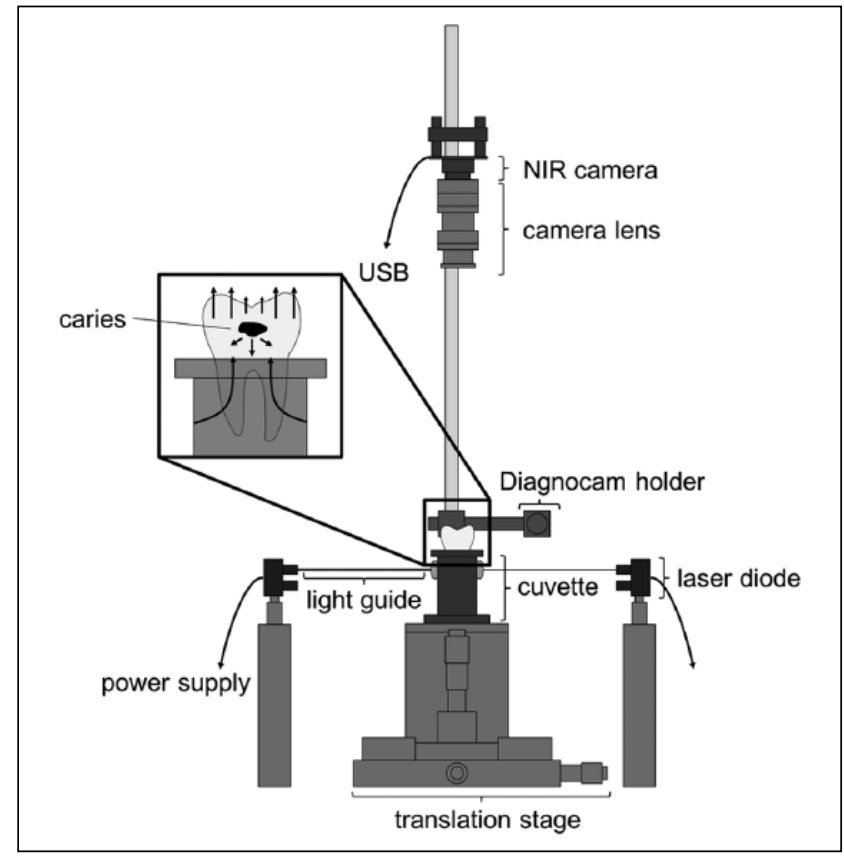

Figure I. Schematic illustration of the near-infrared transillumination setup.

transmitted through a diffusely scattering liquid and guided through the root to the crown. Light scattering by particles in a colloid is known as the Tyndall effect. Different liquids were tested for diffusing and scattering light, including solutions with hydroxyapatite or milk. The best results were obtained using homogenized milk with a $4 \%$ fat content.

A round cuvette with a spill tray was mounted on a vertical and horizontal translation stage with micrometers for fine positioning. Two fiber-optic light guides were inserted through small holes $2 \mathrm{~mm}$ under the rim and subsequently sealed with boxing wax (Kerr). The light guides were connected to a laser optical unit with a 780-nm class 1 laser diode in a housing (KaVo), which is the same laser integrated in the DC. The laser was operated by a $5 \mathrm{~V}$ and $50 \mathrm{~mA}$ laboratory power supply (VLP-1303 PRO, VOLTCRAFT). The cuvette was filled with milk and covered with a $30 \times 30 \mathrm{~mm}$ boxing wax strip with adhered black opaque tape on top to block direct light. A small $10 \times 10 \mathrm{~mm}$ square was cut in the middle, and the resulting recess was covered by self-adhesive foam sealing tape with a small hole in the middle for the teeth. As all investigations were conducted in a darkened room, the only light that could reach the camera was light guided through the root to the crown of the tooth. This was done to standardize the examination and prevent reflections on the tooth surface by ambient light. Images were obtained with an above-mounted NIR camera manufactured by IDS (UI-1241LE-NIR-GL, Imaging Development Systems GmbH) together with a Tamron 50-mm lens (M118FM50, TAMRON Europe GmbH).

In addition, a horizontal arm with a mounting bracket for the DC was attached, so that the DC and the experimental camera could evaluate the same tooth under similar conditions. Instead of the clinical tip of the DC, the laser optical unit of the 


\begin{tabular}{|c|l|}
\hline Index & Description \\
\hline 0 & No tooth decay \\
\hline 1 & Caries visible in enamel \\
\hline 2 & $\begin{array}{l}\text { Caries visible in enamel with } \\
\text { single-point contact to the } \\
\text { dentino-enamel junction }\end{array}$ \\
\hline 3 & $\begin{array}{l}\text { Caries visible in enamel with } \\
\text { extensive contact to the } \\
\text { dentino-enamel junction }\end{array}$ \\
\hline 4 & $\begin{array}{l}\text { Caries visible in enamel and } \\
\text { dentin }\end{array}$ \\
\hline
\end{tabular}

Figure 2. Disease severity scale for use with near-infrared transillumination images. in vitro model was used for transillumination to obtain comparable images. The DC could be pivoted over the tooth, and images obtained with the KaVo KiD (KaVo integrated Desktop) software. Subsequently, an exposure series was shot with the NIR camera with a 4-ms interval between consecutive shots. Images were evenly exposed at $33 \mathrm{~ms}$. Six further over- and underexposed images were taken for an even graduation. The exposure series started at $9 \mathrm{~ms}$ and ended at $57 \mathrm{~ms}$; therefore, a total of 13 images of each tooth were taken. Images with exposure times under $9 \mathrm{~ms}$ or over $57 \mathrm{~ms}$ were not suitable for HDR rendering.

The obtained image sets were further processed with Fiji (Schindelin et al. 2012), a distribution of ImageJ. A weighting factor was calculated for each image (exposure time of the individual image divided by the maximum exposure time of $57 \mathrm{~ms}$ ), and all images merged into a single HDR image using the “KHKsBwHdrCalculatorExt” Plugin for Fiji (K.H.K).

\section{Examiners}

Before assessing the images, 2 experienced examiners (A.L. and F.L.) were given a practical and theoretical training session. Then, all images were analyzed and described using a modified diagnostic classification for NIRT images, describing different stages of proximal caries (0 to 4) (Figure 2) (Söchtig et al. 2014). The assessment of the images was conducted independently by both examiners on a calibrated standard monitor (Windows "Display Color Calibration" wizard) in a darkened room to standardize image evaluation. This was repeated with a minimum interval of $2 \mathrm{wk}$ to prevent misclassification and to calculate the inter- and intrarater reliability.

\section{Validation with Micro-Computed Tomography}

Each tooth was mounted vertically and separately in a water-filled cuvette, which was sealed with Parafilm M (Bemis Company) to stop the samples drying out. The coronal and upper part of the root were scanned using a $\mu \mathrm{CT} 40$ fully shielded, cone-beam, desktop micro-CT scanner (Scanco Medical) at $70 \mathrm{kV}$ and $114 \mu \mathrm{A}$, with a field of view limited to $16.5 \mathrm{~mm}$. The resulting pixel size in the obtained raw data sets (RSQ files) was equal to 0.032 $\mathrm{mm}$. The RSQ files were reconstructed into 3D data sets (ISQ files) and further processed with Fiji (Schindelin et al. 2012) and the plugin "Import ISQ" (K.H.K). Measurements of the horizontal spread of the caries, caries width at the dentino-enamel
Figure 3. Comparison of Diagnocam (DC) and near-infrared transillumination high-dynamicrange imaging (NIRT-HDRI) with $\mu \mathrm{CT}$. 
Table I. Cross-table of Diagnocam and Near-Infrared Transillumination High-Dynamic-Range Imaging (NIRT-HDRI) in Comparison with $\mu$ CT Diagnoses.

\begin{tabular}{|c|c|c|c|c|c|c|c|c|c|c|c|c|}
\hline & & \multicolumn{5}{|c|}{ Diagnocam } & \multicolumn{5}{|c|}{ NIRT-HDRI } & \multirow[b]{2}{*}{ Total } \\
\hline & & 0 & I & 2 & 3 & 4 & 0 & I & 2 & 3 & 4 & \\
\hline \multirow[t]{5}{*}{$\mu \mathrm{CT}$} & 0 & 64 & 3 & I & 0 & 0 & 65 & 3 & 0 & 0 & 0 & 68 \\
\hline & I & 4 & 6 & 0 & I & 0 & 4 & 6 & I & 0 & 0 & 11 \\
\hline & 2 & 3 & 3 & 3 & I & 0 & 3 & 2 & 4 & I & 0 & 10 \\
\hline & 3 & I & I & I & 10 & 4 & I & I & 3 & 7 & 5 & 17 \\
\hline & Total & 72 & 13 & 5 & 12 & 4 & 73 & 12 & 8 & 8 & 5 & 106 \\
\hline
\end{tabular}

Table 2. Sensitivity and Specificity of the Diagnocam and Near-Infrared Transillumination High-Dynamic-Range Imaging (NIRT-HDRI) using Micro-Computed Tomography as Reference Standard.

\begin{tabular}{lccc}
\hline & & Sensitivity & Specificity \\
\hline Diagnocam & Sound & 0.94 & 0.79 \\
& Enamel & 0.57 & 0.93 \\
NIRT-HDRI & Dentin & 0.82 & 0.98 \\
& Sound & 0.96 & 0.79 \\
& Enamel & 0.62 & 0.92 \\
& Dentin & 0.71 & 0.99 \\
\hline
\end{tabular}

junction, and enamel thickness were taken. Measurements were taken 3 times by one examiner, and the mean values calculated. The surfaces were then classified using the following criteria: absence of radiolucency (0), radiolucency in the outer (E1), or the inner half of the enamel (E2), and radiolucency in the dentin (D).

\section{Statistical Analyses}

A crosstabulation was used to compare the DC and NIRTHDRI diagnoses with $\mu \mathrm{CT}$ scores and shows the distribution. The linearly weighted Cohen's Kappa (wк) coefficient was used to measure the inter- and intra-rater reliability for both diagnostic methods and to determine the overall agreement between the DC and NIRT-HDRI results. Agreement values between the DC and NIRT-HDRI assessment per category were calculated using the simple Kappa statistic ( $\kappa)$. To compare $\mu \mathrm{CT}$, DC and NIRT-HDRI on an equivalent basis, a distinction was made for sound surfaces, enamel caries, and dentin caries. For each of these categories sensitivity and specificity were calculated. Data were analyzed using SPSS 23 (SPSS Inc.) and MedCalc Statistical Software 17.5.5 (MedCalc Software bvba).

\section{Results}

Linear wא values for inter-rater agreement showed almost perfect agreement for both diagnostic modalities: 0.9 for the DC and 0.96 for NIRT-HDRI (Landis and Koch 1977). The same applies for intra-rater agreement (A.L. and F.L.): 0.96 and 0.91 for the DC, for NIRT-HDRI 0.9 and 0.93 .

A cross-tabulation (Table 1) presents the distribution of diagnoses. High consistency was noted between DC and NIRT-HDRI diagnoses. A maximum discrepancy of one category was observed for almost all categories. Whereas only a few surfaces were classified by DC and NIRT-HDRI as category 4 (directly associated with dentin caries), many more surfaces were classified as category 4 by $\mu \mathrm{CT}$.

The agreement values $(\kappa)$ between the DC and NIRT-HDRI diagnoses were almost perfect in category $4(0.88)$ and were substantial in categories 0 and 3 ( 0.8 and 0.67$)$. In categories 1 and 2 , the agreement was moderate $(0.59$ and 0.43$)$. The overall agreement (wא) was almost perfect $(0.85 ; 0.95$ confidence interval of 0.78 to 0.92 ).

Thresholds were defined for sound surfaces, enamel caries, and dentin caries. DC or NIRT-HDRI category 0 was classified as sound, category 1 and 2 as enamel caries. The comparison of DC and NIRT-HDRI with $\mu \mathrm{CT}$ (Table 1) showed that most surfaces classified as category 3 had dentin lesions; therefore, category 3 was chosen as the threshold for dentin caries. Next, we calculated the sensitivity and specificity for sound surfaces, enamel caries, and dentin caries (Table 2). The values ranged from 0.57 to 0.99 , and were similar for both methods in the different categories. NIRT-HDRI had a higher sensitivity for sound surfaces and enamel caries as well as a higher specificity for dentin caries. The lowest sensitivity was calculated for enamel lesions, 0.57 for DC and 0.62 for NIRT-HDRI; however, the specificity was high.

The generated NIRT-HDR images show more details and brightness gradations in shadows and light than conventional NIRT images (Figure 3). Under- and overexposed areas could be reduced, and caries differentiated more clearly in most cases.

\section{Discussion}

The early detection of incipient proximal caries lesions is of increasing importance because, at present, these initial lesions can be treated by non- and micro-invasive methods to prevent their progression onto stages where it is necessary to "drill and fill" (Dorri et al. 2015). Visual inspection and radiographic examinations often underestimate proximal caries lesions (Bader et al. 2001; Selwitz et al. 2007); for this reason, alternatives, such as transillumination, need to be investigated and validated.

This study evaluated the suitability of NIRT combined with HDRI for the detection of proximal caries lesions. The project focused on the in vitro comparison of proximal caries diagnostics with the DC and NIRT-HDRI using $\mu \mathrm{CT}$ as reference standard. It was not possible for us to obtain a satisfactory image 
quality when using the DC with the clinical tip in vitro without scattering the laser light. This resulted in overexposed image areas and speckle phenomena. With a light diffusing liquid, we could simulate the scattering properties of the tissue surrounding the tooth. To generate comparable images with both diagnostic methods, the original DC lasers from Kavo were used as a common light source for both methods. Although this differs to that used in the clinic, it was necessary for our in vitro model.

In dentin, the scattering coefficient showed minimal decreases with the increasing wavelength but was very low in enamel in the NIR range (Kienle et al. 2006; Moritz 2006). This implies an increasing penetration depth of light, which is needed for transillumination (Fried et al. 2005). The optimal wavelength for NIRT is between $795 \mathrm{~nm}$ and $\sim 1,600 \mathrm{~nm}$ (Darling et al. 2006). Transilluminating teeth for caries detection in this range was patented in 2006; therefore, manufacturers have used slightly lower wavelengths for their products (for example, $780 \mathrm{~nm}$ ).

The correlation of DC and NIRT-HDRI diagnoses showed a strong agreement for categories 0 and 4 , representing sound teeth and dentin caries ( $\kappa 0.80$ and 0.88 ), whereas other categories showed less agreement. These differences in diagnoses may have been caused by several factors. First, the $\mu \mathrm{CT}$ validation showed that the selected samples contained more sound than decayed surfaces; at least, the demineralization in these samples were superficial and not detectable with any of the methods used. Therefore, discordant diagnostic decisions did not have a strong effect in category 0 . Furthermore, the diagnosis "sound" is usually a clear decision for examiners (Bader et al. 2001), whereas other categories are more subjective, requiring interpretation and judgment, especially to allocate between borderline cases; for example, when deciding whether a carious lesion has single-point contact to the dentino-enamel junction (category 2 ) or is located immediately before the junction (category 1). Categories 1 and 2 showed low agreement values in our study ( $\kappa 0.59$ and 0.43 ). In addition, categorization may be influenced in some cases by the camera angle. It may be worth combining categories 1 and 2 in future studies. However, it must first be examined whether it makes sense to combine these categories or whether another classification system should be established. Taken together, this information might explain the moderate Kappa values for both categories, and highlights a universal problem in the visual classification of borderline cases using systems as compared to continuous scales.

In radiography, the pulp serves as a reference to evaluate lesion depth; this is not possible with NIRT due to the superposition of scattering by mineral crystals, collagen fibrils, and dentinal tubules in dentin (Zijp and ten Bosch 1993). Likewise, caries in dentin can only be shown in some cases. Different dentin lesions with similar extension in X-ray images are not always visible in NIRT images because of visibility. We suggest that clear visualization of dentin caries (category 4) is only possible if the lesion is located directly under the occlusal enamel layer. More cervically-located dentin lesions - with healthy dentin between the lesion and occlusal enamel- cannot be visualized. In addition, the extension of the caries and the degree of demineralization affect visualization. Where there is high contrast between the demineralization and the sound enamel, it is possible to indirectly extrapolate a dentin lesion from caries visible in the enamel with extensive contact to the dentino-enamel junction (Kühnisch et al. 2016). These findings should be verified histologically or by computer tomography in further studies.

In our study, we preferred $\mu \mathrm{CT}$ as a reference standard, as it offers several advantages. Without destroying the samples, even small structural changes can be visualized. By taking scans of the entire sample, we can determine where the lesion is most advanced. $\mu \mathrm{CT}$ has been used in other studies for examination and validation (Matsuda 2002; Shahmoradi and Swain 2017), and is reported to perform equal or better than histology for in vitro caries assessment (Soviero et al. 2012; Özkan et al. 2015). Using $\mu \mathrm{CT}$ for validation, we found no major differences in terms of sensitivity or specificity between the 2 methods. The lower sensitivity for enamel caries may be explained by the fact that small enamel demineralization not visible with the Diagnocam or NIRT-HDRI were detectable in $\mu \mathrm{CT}$ images and thus rated as enamel caries. The threshold chosen for dentin caries, category 3 , seems to be appropriate, as sensitivity and specificity are high in this category; as already described above, category 4 is not a suitable threshold for dentin caries.

The combination of NIRT and HDRI allows for a greater range of luminance levels using HDRI. Studies in other disciplines, for example, architecture, have shown the utility of HDRI for identifying detail (Kontogianni et al. 2015). Underand overexposed image areas can be reduced, especially compared to clinical images. It must be acknowledged, however, that in the present study, NIRT was performed under optimal conditions, and direct light to the camera was blocked. In daily clinical practice, this is usually not possible. In such situations, we would expect HDRI to be superior to simple exposure images. One potential disadvantage of HDRI in clinical use could be blurred images due to the summation of exposure times of the image series and no rigid fixation between the camera and tooth. Possible solutions could be a rigid mechanical support or software-based image stabilization.

To sum up, we successfully developed an in vitro model that is well-suited for validating the Diagnocam and NIRT. Our in vitro study revealed that both methods seem to be well-suited for proximal caries detection. Obtaining images suitable for diagnostics is more difficult on patients than in vitro; therefore, the benefits of HDRI may be more apparent in the clinical setting. This should be evaluated in future studies. Distinguishing between processed demineralized enamel and dentin lesions seems to be a specific problem for NIRT and cannot be balanced using HDRI.

\section{Author Contributions}

A. Lederer, F. Litzenburger, contributed to conception, design, data acquisition, analysis, and interpretation, drafted and critically revised the manuscript; K.H. Kunzelmann, contributed to 
conception, design, data analysis, and interpretation, critically revised the manuscript; R. Hickel, contributed to data acquisition, critically revised the manuscript. All authors gave final approval and agree to be accountable for all aspects of the work.

\section{Acknowledgments}

This work was supported by the Department of Operative Dentistry and Periodontology of the University Hospital of the LMU Munich in Germany. The authors declare no potential conflicts of interest with respect to the authorship and/or publication of this article.

\section{References}

Abdelaziz M, Krejci I. 2015. Diagnocam-a near infrared digital imaging transillumination (nidit) technology. Int J Esthet Dent. 10(1):158-165.

Abdelaziz M, Rizzini AL, Bortolotto T, Rocca GT, Feilzer AJ, Garcia-Godoy F, Krejci I. 2016. Comparing different enamel pretreatment options for resin-infiltration of natural non-cavitated carious lesions. Am J Dent. 29(1):3-9.

Abesi F, Mirshekar A, Moudi E, Seyedmajidi M, Haghanifar S, Haghighat N, Bijani A. 2012. Diagnostic accuracy of digital and conventional radiography in the detection of non-cavitated approximal dental caries. Iran J Radiol. 9(1):17-21.

Bader JD, Shugars DA, Bonito AJ. 2001. Systematic reviews of selected dental caries diagnostic and management methods. J Dent Educ. 65(10):960-968.

Bussaneli DG, Restrepo M, Boldieri T, Pretel H, Mancini MW, Santos-Pinto L, Cordeiro RC. 2015. Assessment of a new infrared laser transillumination technology $(808 \mathrm{~nm})$ for the detection of occlusal caries-an in vitro study. Lasers Med Sci. 30(7):1873-1879.

Darling CL, Huynh GD, Fried D. 2006. Light scattering properties of natural and artificially demineralized dental enamel at $1310 \mathrm{~nm}$. J Biomed Opt. 11(3):34023.

Dorri M, Dunne SM, Walsh T, Schwendicke F. 2015. Micro-invasive interventions for managing proximal dental decay in primary and permanent teeth. Cochrane Database Syst Rev. 11:CD010431.

Featherstone JD, Young D. 1999. The need for new caries diagnostic detetion methods. Lasers in Dentistry V, Proc. SPIE 3593:134-140.

Fried D, Featherstone JD, Darling CL, Jones RS, Ngaotheppitak P, Buhler CM. 2005. Early caries imaging and monitoring with near-infrared light. Dent Clin North Am. 49(4):771-793, vi.

Hume WR. 1993. Need for change in standards of caries diagnosis-perspective based on the structure and behavior of the caries lesion. J Dent Educ. 57(6):439-443.

Jones R, Huynh G, Jones G, Fried D. 2003. Near-infrared transillumination at $1310-\mathrm{nm}$ for the imaging of early dental decay. Opt Express. 11(18): 2259-2265.

Kachalia P. 2015. Caries detection using near-infrared transillumination: Adding objectivity to diagnosis to save tooth structure. Dent Today. 34(5):86-88.
Kienle A, Michels R, Hibst R. 2006. Magnification-a new look at a long-known optical property of dentin. J Dent Res. 85(10):955-959.

Kierzek A. 1995. Diaphanoscopy (transillumination) of maxillary sinuses: A method too early forgotten? Otolaryngol Pol. 49(4):371-379.

Kontogianni G, Stathopoulou EK, Georgopoulos A, Doulamis A. 2015. Hdr imaging for feature detection on detailed achitectural scenes. The International Archives of the Photogrammetry, Remote Sensing and Spatial Information Sciences. XL-5/W4. [Accessed on 1/29/2018] Available from: http://adsabs.harvard.edu/abs/2015ISPAr.XL5..325K.

Kühnisch J, Söchtig F, Pitchika V, Laubender R, Neuhaus KW, Lussi A, Hickel R. 2016. In vivo validation of near-infrared light transillumination for interproximal dentin caries detection. Clin Oral Investig. 20(4):821-829.

Landis JR, Koch GG. 1977. The measurement of observer agreement for categorical data. Biometrics. 33(1):159-174.

Matsuda Y, Hanazawa T, Seki K, Araki K, Okano T. 2002. Comparison between rvg ui sensor and kodak insight film for detection of incipient proximal caries. Oral Radiol. 18(2):41-47.

Moritz A. 2006. Orale lasertherapie. Berlin: Quintessenz Verlag. p. 197-198.

National Institutes of Health. 2001. Diagnosis and management of dental caries throughout life: Nih consensus statement. Washington (DC): National Institutes of Health.

Özkan G, Kanli A, Baseren NM, Arslan U, Tatar I. 2015. Validation of microcomputed tomography for occlusal caries detection: An in vitro study. Braz Oral Res. 29(1):S1806-83242015000100309.

Poorterman JH, Aartman IH, Kalsbeek H. 1999. Underestimation of the prevalence of approximal caries and inadequate restorations in a clinical epidemiological study. Community Dent Oral Epidemiol. 27(5):331-337.

Poorterman JH, Aartman IH, Kieft JA, Kalsbeek H. 2002. [clinical underestimation of the prevalence of approximal dentin lesions and inadequate restorations]. Ned Tijdschr Tandheelkd. 109(2):47-50.

Schindelin J, Arganda-Carreras I, Frise E, Kaynig V, Longair M, Pietzsch T, Preibisch S, Rueden C, Saalfeld S, Schmid B, et al. 2012. Fiji: An opensource platform for biological-image analysis. Nat Methods. 9(7):676-682.

Selwitz RH, Ismail AI, Pitts NB. 2007. Dental caries. Lancet. 369(9555):51-59.

Shahmoradi M, Swain MV. 2017. Micro-ct analysis of naturally arrested brown spot enamel lesions. J Dent. 56:105-111.

Shurtleff D, Foltz E, Fry D. 1966. Clinical use of transillumination. Arch Dis Child. 41(216): 183-187.

Simon JC, Lucas S, Lee R, Darling CL, Staninec M, Vanderhobli R, Pelzner R, Fried D. 2015. In vitro near-infrared imaging of natural secondary caries. Proc SPIE Int Soc Opt Eng. 9306. pii: 93060F.

Simon JC, S AL, Lee RC, Darling CL, Staninec M, Vaderhobli R, Pelzner R, Fried D. 2016. Near-infrared imaging of secondary caries lesions around composite restorations at wavelengths from 1300-1700-nm. Dent Mater. 32(4):587-595.

Söchtig F, Hickel R, Kühnisch J. 2014. Caries detection and diagnostics with near-infrared light transillumination: Clinical experiences. Quintessence Int. 45(6):531-538.

Soviero VM, Leal SC, Silva RC, Azevedo RB. 2012. Validity of microct for in vitro detection of proximal carious lesions in primary molars. J Dent. 40(1):35-40.

Zijp JR, ten Bosch JJ. 1993. Theoretical model for the scattering of light by dentin and comparison with measurements. Appl Opt. 32(4):411-415. 